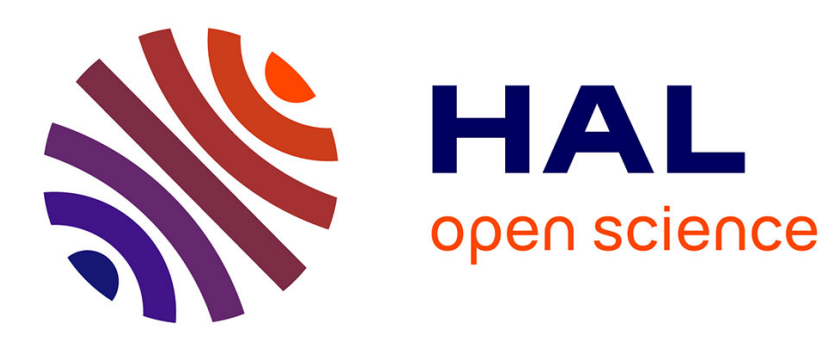

\title{
Estimating Illumination Direction of 3D Surface Texture Based on Active Basis and Mojette Transform
}

Junyu Dong, Liyuan Su, Yu Zhang, Florent Autrusseau, Yang Zhanbin

\section{To cite this version:}

Junyu Dong, Liyuan Su, Yu Zhang, Florent Autrusseau, Yang Zhanbin. Estimating Illumination Direction of 3D Surface Texture Based on Active Basis and Mojette Transform. Journal of Electronic Imaging, 2012, 21 (1), pp.013023-1. 10.1117/1.JEI.21.1.013023 . hal-00674451

\section{HAL Id: hal-00674451 https://hal.science/hal-00674451}

Submitted on 17 Apr 2012

HAL is a multi-disciplinary open access archive for the deposit and dissemination of scientific research documents, whether they are published or not. The documents may come from teaching and research institutions in France or abroad, or from public or private research centers.
L'archive ouverte pluridisciplinaire HAL, est destinée au dépôt et à la diffusion de documents scientifiques de niveau recherche, publiés ou non, émanant des établissements d'enseignement et de recherche français ou étrangers, des laboratoires publics ou privés. 


\title{
Estimating Illumination Direction of 3D Surface Texture
}

\section{Based on Active Basis and Mojette Transform}

\author{
Junyu Dong, ${ }^{1, *}$ Liyuan Su, ${ }^{1}$ Yu Zhang, ${ }^{1}$ Florent Autrusseau ${ }^{2}$ Zhanbin Yang ${ }^{1}$ \\ ${ }^{1}$ Department of Computer Science and Technology, Ocean University of China, Qingdao, China \\ ${ }^{2}$ LUNAM Université, Université de Nantes, IRCCyN UMR CNRS 6597, Polytech \\ Nantes, rue Christian Pauc BP 5060944306 Nantes, France \\ *Corresponding author: dongjunyu@ouc.edu.cn
}

\begin{abstract}
This paper describes an approach that, given an image of a rough surface texture, can estimate the direction from which the surface was lit. Unlike previous work, we require neither surface isotropy nor that the texture is included in the training set. The approach is based on Active Basis and the Mojette transform. The Mojette transform is used to estimate the orientation of the texture; it does so by finding which training samples have similar orientation features. The Active Basis model is then learned from training images by the shared pursuit algorithm. Next, the base histograms of the test image and textures with similar orientation features in the training set are compared so that the illumination directions can be estimated by minimizing their correlation coefficients.
\end{abstract}

\section{Introduction}

Many visual textures are formed by rough surfaces. The appearance of these textures is affected by the direction from which they are lit-illumination can be modeled as a directional 
filter [11]. Isotropic surface textures will form directional textures whose directionality is aligned with the lighting vector. For anisotropic surfaces the situation is more complex: the illumination will either accentuate or attenuate the underlying surface directionality and the directionality of the resulting image will depend on both illumination and surface directionality. Estimating the direction from which a rough surface is lit is an important task. Most work has assumed the surface texture is isotropic; this paper addresses the more difficult task of estimating the illumination direction for anisotropic surfaces.

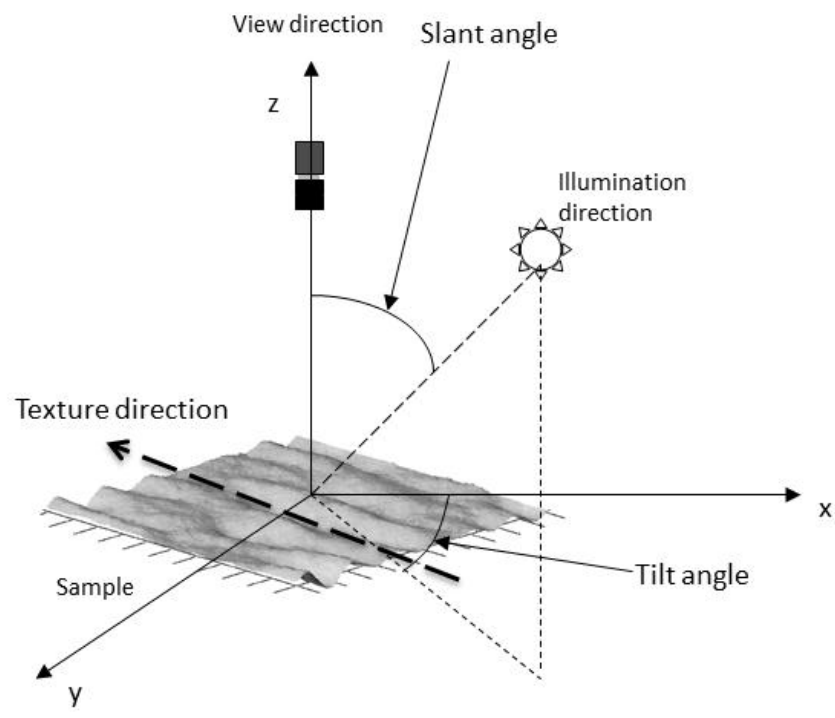

(a)
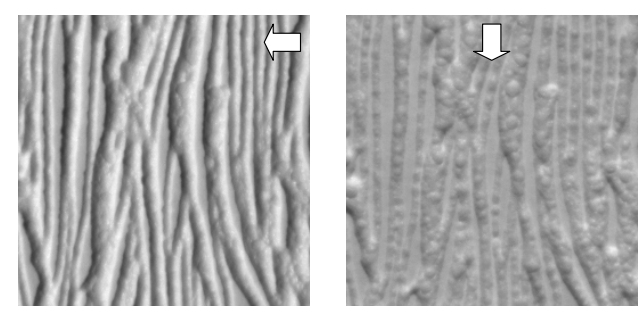

(b)

Fig.1. (a) Definition of texture direction and illumination direction. (b) Two images of a 3D surface texture illuminated from different directions. The block arrows show the illuminant directions. 
When an anisotropic texture is lit from a given direction, surface bumps that are oriented towards the light become brighter and form edge-like structures, Fig.1. Our approach uses these structures to account for the surface directionality and to estimate the direction of the light source.

Recently, Wu et al. [1] proposed an active basis model for learning deformable templates from image patches of various object categories. The technique has been used for object detection and recognition [2,3]. An active basis consists of a small number of Gabor wavelet elements at different locations and orientations. These elements are generated from training images by finding the maximum responses of Gabor filters and essentially form the "sketch" of the training samples. The elements are also allowed to slightly perturb their locations and orientations, so that they can be well combined to represent each individual training or testing example. When a surface texture is anisotropic, due to the strong response between surface structures and the light, "edge" information containing implicit illumination directions can be accurately modeled by active basis. Once we capture this information, we can easily estimate the illumination direction with the Active Basis model.

To implement the method, the Mojette transform is employed to measure the orientations of surface texture images. In the context of this paper, we use the term "texture orientation" to refer to as the angle of the predominant direction or directional trend of texture patterns. As in literature $[13,21]$, we also use the term "texture direction". Our method consists of two stages: the texture direction is first estimated by applying the Mojette transform to the image. Texture direction features derived from the Mojette transform are then used to find textures with similar orientations to the test one. Then the Active Basis model is employed to extract illumination direction features. The Active Basis model can be learned from training image patches by the 
shared pursuit algorithm [4]. For a test texture, the histogram of its active basis is compared with those of the training models, and the illumination direction of the test texture is determined by the sample with the most similar histogram. The scheme of our algorithm is illustrated in Fig.2.

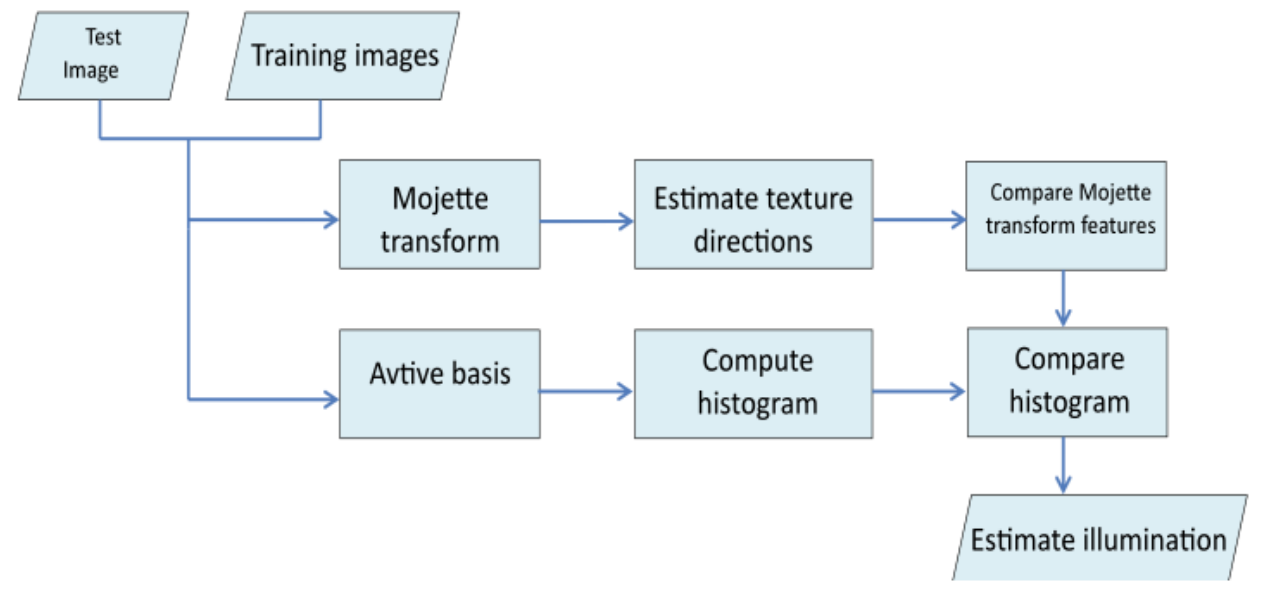

Fig.2. Flowchart of the proposed method

Our contribution in this paper is a method that is able to estimate illuminant's azimuthal angles from an image of a surface texture. The method is based on active basis and the Mojette transform, and the test sample is not required to be included in the training set.

In the next section, we review previous works in the area of illumination estimation and texture direction measurement. Section 3 describes the orientation estimation using Mojette transform on 3D anisotropic surface texture. Next, in section 4 we present in details the Active Basis model and the estimation of illumination directions. The results produced by our method are presented in section 5. Finally, in section 6 we draw our conclusions and discuss limitations and future works.

\section{Previous Work}

Estimating the direction of illumination 
Early studies on illumination direction estimation $[5,6]$ were based on techniques from Shape from Shading. Severe constraints had to be imposed in order to find a reasonable solution $[7,8]$. Koenderink and Pont [7] developed a theory based on second order statistics for estimating the illuminant's azimuth from a single image formed under the Lambertian model. Their algorithm imposes several restrictions: the tested surface has to be isotropic, Gaussian and have constant albedo.

Varma and Zisserman [8] extended this method to deal with surfaces having a spatially varying albedo. Although this approach could recover the azimuthal angle more accurately, this estimation mechanism failed for strongly anisotropic surfaces.

Karlsson et al. [9] presented a theory of combining both the gradient-based and the Hessian-based tensors to estimate illumination directions when dealing with the case of small amounts of surface anisotropy. They tested their theoretical predictions on computer-rendered Gaussian surfaces. As a result, estimates were accurate if the parameters are close to their assumed values. With the real world textures in [10], due to the non-Lambertian, higher-relief, oblique viewing or image noise, error was amplified by the multiplicative factors introduced in the theory. This gave rise to more unstable estimation with an average error of $17.2^{\circ}$ [9].

Chantler et al. [11,12] proposed an approach to identify illuminant direction and classify textures. A probabilistic model was developed using an existing model of the dependency of texture features on lighting directions. The most likely illumination vector could be estimated by optimizing the model. The technique accurately estimates the azimuth of the light source. However, it does require more training and computation than a conventional classifier.

\section{Measuring the Direction of Visual Textures}


The Radon transform was used by Jafari-Khouzani and Soltanian-Zadeh [13] to detect the principal directions of the texture. They considered that the variance of the projection presents a local maximum at the principal orientations. In practice, the method is unsuitable for complex textures, e.g. textures having straight lines along several directions.

Jia et al. [14] later introduced the Mojette transform to estimate texture directions. They performed Mojette transform on a set of textures lit from different tilt angles. Unlike [13], a threshold is defined to provide the candidate orientations for complex textures which may have several directions. They also estimated the illumination tilt angle with the variances of Mojette projections. However, the algorithm can only estimate illumination directions of textures included in the training image sets. In this paper, both the texture orientation and the illumination direction are estimated from the model.

In this paper we address the problem of estimating the illuminant's azimuthal angles from images of anisotropic textured surfaces. We can estimate the illumination direction of a texture which is not included in the training data set but which has a similar orientation to a sample in the training set. Finally, if no similar texture exists within the training set, our model can provide the detected main orientation(s). In this case, the illumination direction of the test texture can also be predicted.

\section{Texture Direction Estimation}

We use the Mojette transform to compute the texture directions, which is a clue for illumination estimation. Unlike previous work on estimating 2D texture orientations in [13], we determine the texture directions by using the Mojette transform, which is an exact and discrete form of Radon transform [15] defined for specific projection angles. This scheme can be effectively integrated in the later stage to estimate illumination directions of 3D surface textures. 


\section{Mojette Transform}

Due to its simple mathematical framework, the Mojette transform is widely used in images analysis $[16,17]$. Like the classical Radon transform, the Mojette transform represents the image as a set of projections (as few as 1 depending on the angle set). However, the Mojette transform has an exact inverse from a finite number of discrete projections. The rational projection angles are defined by a set of discrete vectors $\left(p_{i}, q_{i}\right)$ as $\theta_{i}=\tan ^{-1}\left(q_{i} / p_{i}\right)$. These vector must respect the condition that $p_{i}$ and $q_{i}$ are coprime (i.e. $\left.\operatorname{gcd}\left(p_{i}, q_{i}\right)=1\right)$, and since it is $\pi$-periodic, $q_{i}$ must be positive except if $\left(p_{i}, q_{i}\right)=(1,0)$. The transform domain of an image is a set of projections where each element (called a "bin" as in tomography) corresponds to the sum of the pixels centered on the line of projection. The "Dirac" Mojette transform is defined for each projection angle by the operator:

$$
\begin{gathered}
{\left[\mathrm{M}_{\delta} f\right](b, p, q)=\operatorname{proj}_{\delta}(b)=\sum_{k=-\infty}^{+\infty} \sum_{l=-\infty}^{+\infty} f(k, l) \Delta(b+k q-p l)} \\
M_{p, q} f(k, l)=\sum_{k=-\infty}^{+\infty} \sum_{l=-\infty}^{+\infty} f(k, l) \Delta(b+q k-l p)
\end{gathered}
$$

where $(k, l)$ defines the location of an image pixel, $b$ is the index of the bin on a given $(p, q)$ projection and $\Delta(n)$ is the Kronecker Dirac function equal to 1 when $n=0$ and zero otherwise, or, in other words, $\Delta(n)=\left\{\begin{array}{ccc}1 & \text { if } & n=0 \\ 0 & \text { if } & n \neq 0\end{array}\right.$. The equation $b=-k q+p l$ represents the line of projection (the set of projected pixels). Thus, $\Delta(b-k q+p l)$ is equal to 1 only for those pixels. The Mojette transform, $M_{I} f(k, l)$ corresponds to the set of $I$ projections as 
$M_{I} f(k, l)=\left\{M_{p_{i}, q_{i}} f ; i \in 1,2 \ldots I\right\}$. The number of bins, $B_{i}$, for each projection depends on the chosen direction vector $\left(p_{i}, q_{i}\right)$, and for a $P \times Q$ image is found as

$$
B_{i}=(Q-1)\left|p_{i}\right|+(P-1) q_{i}+1
$$

The "Dirac" Mojette transform described above, considers each pixel as a Kronecker Dirac function, however, in the following, the "Haar" Mojette transform was used. The "Haar" Mojette transform (sometimes named spline 0 Mojette transform) considers every pixel as a unit square, and basically computes a convolution on the projections; the convolution kernel depends on the projection angle. This gives some advantages over the simple Dirac Mojette Transform. Interested readers should refer to [19] for details on the Haar Mojette transform.

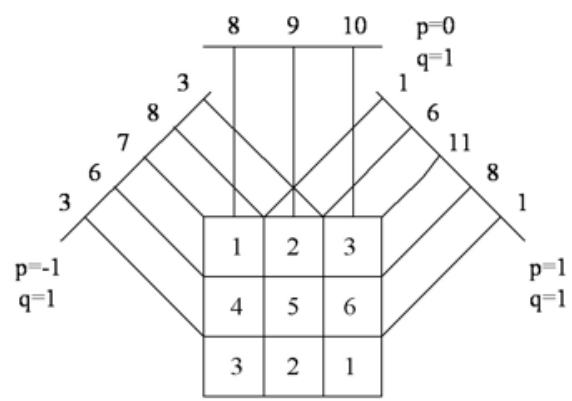

Fig. 3.Mojette transform of a $3 \times 3$ example image.

Fig. 3 shows the Dirac Mojette Transform for a $3 \times 3$ image and for the set of directions $S=\{(-1,1),(1,1),(0,1)\}$ giving 13 bins. The algorithmic complexity of the Mojette transform for an image with projections is $O(P Q I)$.

\section{Texture Direction Estimation}


We use the Mojette transform to detect directional trends in 3D surface texture images. The principal direction of a surface texture can be roughly described as the direction along which there are more approximately straight lines. The Mojette transform along this direction usually has larger variations. Therefore, the variance of the projection at this direction is the local maximum $[13,14]$. Fig. 4 below shows the Mojette projections for two distinct textures. The top panel (Fig. 4(a)) depicts 7 Mojette projections (corresponding to the angles: $0^{\circ}, 23^{\circ}, 45^{\circ}, 75^{\circ}$, $90^{\circ}, 135^{\circ}$, and $172^{\circ}$ ) for the texture "acd" of the PhoTex database. This texture exhibits two main orientations $\left(0^{\circ}\right.$ and $\left.90^{\circ}\right)$; as we can see from this figure, the Mojette projections for these two orientations present the highest variations.

On the center panel (Fig. 4(b)), the same figure is given for an isotropic texture (Texture "aap" from the PhoTex database). For such a scenario, we can notice that all projections present a quite noisy but flat distribution with significantly lower variances. Finally, the lower panel (Fig. 4(c)) represents the input images ("acd" and "aap") along with their Radon sinograms, in order to clearly show the different representations between the Mojette transform and the Radon transform.

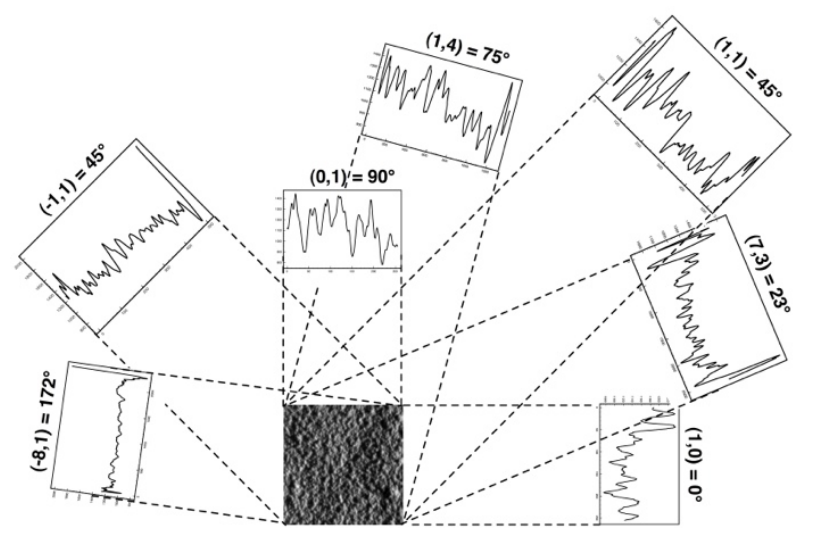

(a) 


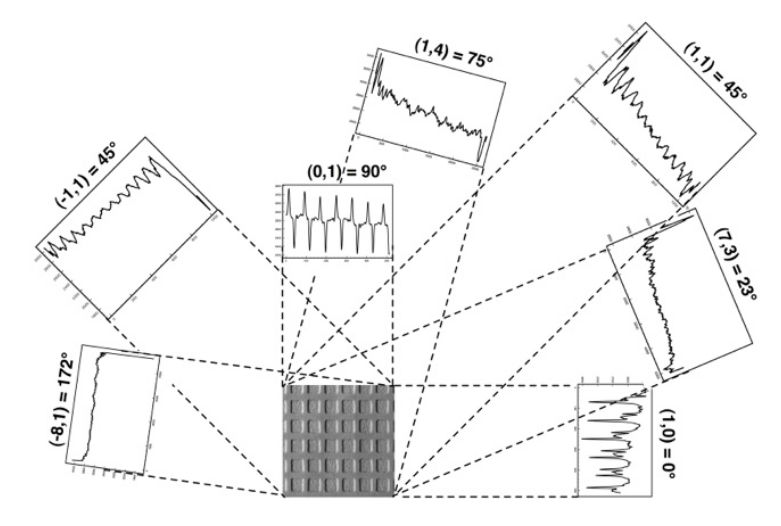

(b)
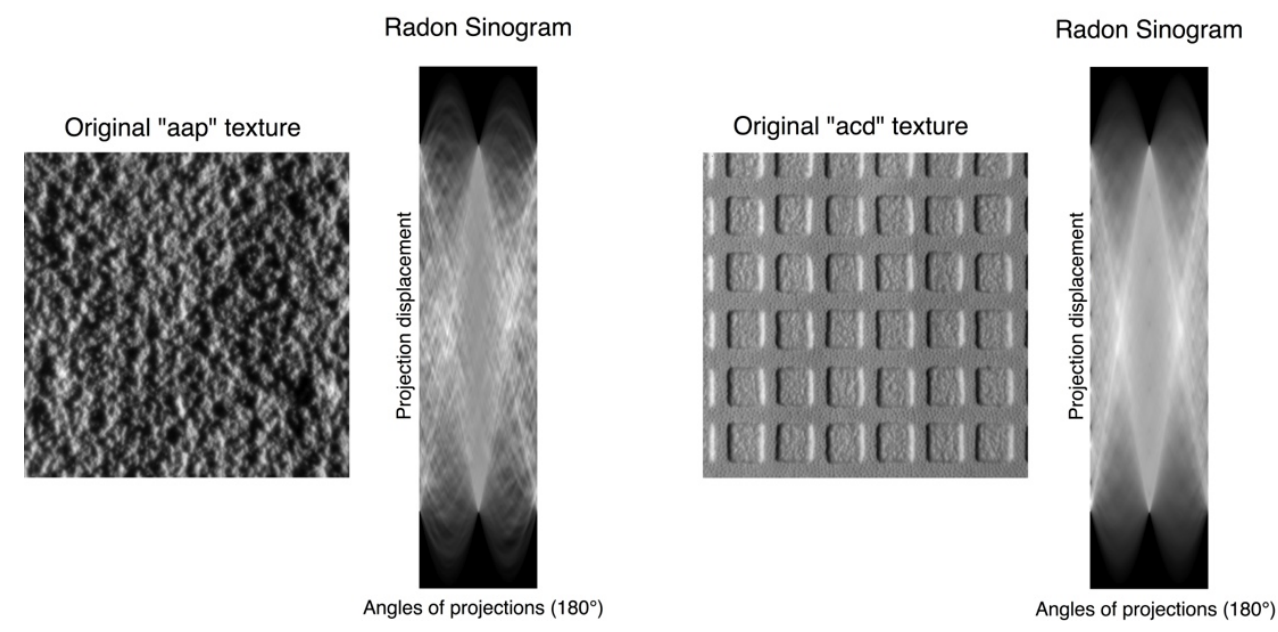

(c)

Fig. 4. Some examples of Mojette projections on two texture images, as well as the Radon sinogram.

For each image in one surface set, we compute $N_{\text {proj }}=72$ Mojette projections with projection angle from $0^{\circ}$ to $180^{\circ}$. Then we calculate the variance of each projection. A $N_{\text {proj }}{ }^{-}$ dimension column vector $V$ is derived from each image.

As discussed before, it is obvious that the variance of the Mojette projection reaches its peaks when the projection direction is parallel to the texture orientation and is lower for other texture orientations. 
We first normalize the variance with respect to the number of projection bins, as they are different across different projection angles. Then the variance vector is normalized by dividing each element by $\sum_{i=1}^{N_{\text {proj }}} V_{i}$. As in [13], we calculate the second derivative of $V$, denoted as $V^{\prime \prime}$. The principal surface texture direction is detected when $V^{\prime \prime}$ reaches its minimum value. Fig. 5 shows two example sets of vectors $V$ and $V^{\prime \prime}$ of surface textures labeled as "acd" and "ace" from the PhoTex database [10]. The x-axis being the orientation angle of the Mojette projection, and the y-axis respectively stands for $V$ (second column) and $V^{\prime \prime}$ (third column).

With vector $V^{\prime \prime}$, the directional similarity between two texture images can be calculated. Our goal is to estimate the illumination direction of a given texture; however, literature shows that it is difficult to accurately obtain this information from a single image [8]. We therefore employ a training set of texture samples captured under known illumination directions. We first find a set of textures having similar orientations as the input test image by comparing the Mojette transform vectors $V^{\prime \prime}$, and then we can estimate its illumination direction by matching illumination features, i.e. the histograms of the active basis, between the test image and the images with similar orientation in the training set. However, the novel point of this paper is that even if the test surface texture is not included in the training data, we can still obtain similar texture directions by comparing $V^{\prime \prime}$ between test and training images. We can then use the training samples with similar texture directions to estimate the illumination direction of the test surface. Experimental results and estimated directions are shown in section 5.

It should be noted that if there is no training or testing datasets, the model can simply output main texture orientations for every given image. 

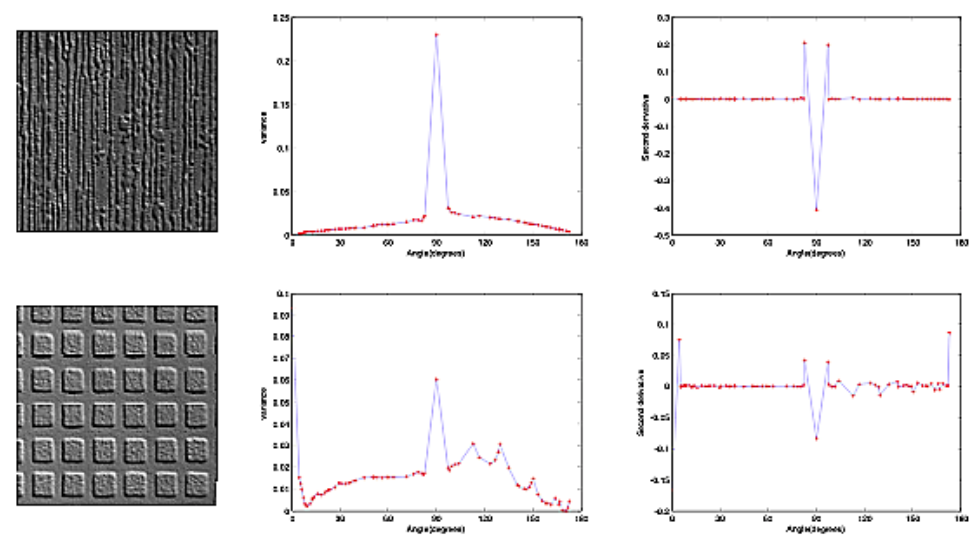

Fig. 5. Plots of $\mathrm{V}$ (middle column) and V" (right column) of surface "ace" (left-top) and "acd" (left-bottom). The plot of $\mathrm{V}$ " shows that $90^{\circ}$ is the main orientation of surface "ace", whereas $0^{\circ}$ and $90^{\circ}$ are the main orientations of surface "acd".

\section{Illumination Direction Estimation}

Given a test image with an unknown illumination direction, we first calculate its texture orientation vector with the Mojette transform. The orientation vector is then used to find textures having similar orientations to the test sample. Then, active basis features are extracted so that the base histograms can be compared for estimating illumination directions of the test image. This section describes in details the Active Basis model learning and illumination direction estimation.

\section{Feature Extraction for Active Basis}

$\mathrm{Wu}$ et al. [1] proposed the Active Basis model for deformable templates to share sketches, where each sketch is allowed to shift in position and orientation. An active basis consists of a small number of Gabor wavelet elements at different locations and orientations, and these elements are allowed to slightly perturb their locations and orientations before they are linearly combined to generate each individual training or testing example. For example, if we can learn an active base model for a certain car from a set of images, we may use the active basis to 
model other cars by varying the basis' locations and orientations. The model learning procedure will be described in more details in the next section.

However, the Gabor filters employed by the original algorithm for extracting objects' features can be computationally very expensive. Instead, we use the Haar feature to improve efficiency. Unlike the convolution with Gabor filter, these rectangle features can be computed very rapidly using an intermediate representation for the image, called the integral image [18].

Haar features are composed of two or more regions, which have the same size and shape and are horizontally or vertically adjacent. It is defined as:

$$
\text { feature }_{I}=\sum_{i \in I=\{1, \ldots, N\}} \omega_{i} \operatorname{RecSum}\left(r_{i}\right)
$$

where $(1, \ldots, N)$ denote the number of rectangles that feature $I$ comprises, and $\omega_{i}$ is the weight value of the $i$ th rectangle. $\operatorname{RecSum}\left(r_{i}\right)$ is the sum of all pixels within the $i$ th rectangle.

In this paper, we expand the Haar features to a set of Active Haar features and improved edge features. Suppose $N$ is the number of the orientation that the edge features represent; the orientation of edge features takes $N$ equally spaced angles in $[0, \pi]$. Fig. 6 shows our improved Active Haar with $N=15$. The features represented with Active Haar are then employed for training models in the following section.

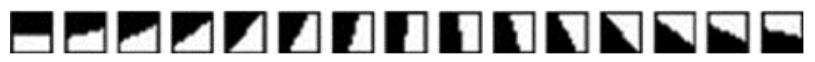

Fig. 6. Improved Active Haar with $\mathrm{N}=15$.

\section{Model Training}

In [4], Wu et al. developed an active sketch algorithm which can learn an object template with very intuitive sketches from image patches of various object categories. The algorithm 
selects the elements of the active basis sequentially from a dictionary of Gabor wavelets. When an element is selected at each step, a perturbed version of this element is added to improve the encoding of each example. Our model training process can be seen as a variation of the original model. Since each surface texture is represented by multiple images captured under different illumination directions, we generate the active basis model for each image separately and all bases forms the final model. Fig. 7 shows the base models of texture "abj" in [10]. The whole process comprises the following three steps:

(1) For each image, compute the Haar response $B_{m, i}$ for each base;

(2) Select an active base according to the shared sketch algorithm [1]. The general idea is to allow the Active base $B_{i}$ to shift its location along its normal direction and also allow it to shift its orientation to minimize $\left\|I-\sum_{i=1}^{n} c_{i} B_{i}\right\|$, where $B_{i}$ can be expressed as

$$
\begin{gathered}
B_{i}=B_{x_{i}, y_{i}, s, \alpha_{i}}, i=1, \ldots, n \\
x_{i}=x_{i}+\delta_{i} \sin \alpha_{i}, \\
y_{i}=y_{i}+\delta_{i} \cos \alpha_{i}, \\
\alpha_{i}=\alpha_{i}+\gamma_{i} \\
\delta_{i} \in\left[-b_{1}, b_{1}\right], \gamma_{i} \in\left[-b_{2}, b_{2}\right]
\end{gathered}
$$

Where $n$ is the number of Active Haar, $\left(x_{i}, y_{i}\right)$ is the base location coordinate, $\alpha_{i}$ is the base angle, and $\left[b_{1}, b_{2}\right]$ are the range for allowed translation and rotation;

(3) iterate step (2), until a fixed number of bases are selected. 

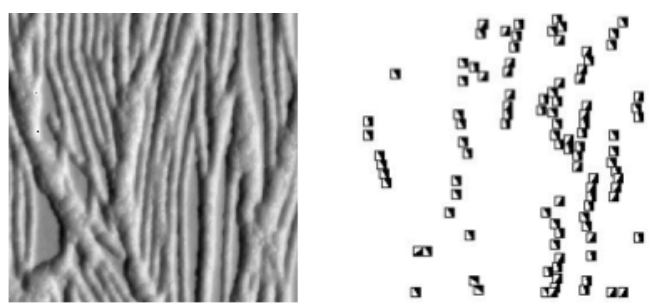

Fig. 7. The base model for texture "abj" with the illumination tilt angle of 0 . Left: the original sample; Right: the extracted base model. The number of orientations in the Haar feature is 9 and the basis number is 100.

After applying this process to each image, we can generate a base model. Then we calculate a base histogram of each image by counting how many times each orientation of base has been selected within the corresponding model. Fig. 8 shows the models and base histograms of texture "acd" from the PhoTex texture database [10].

Previous work in the literature has shown that "edge count" can be used for texture direction analysis and texture classification; some theoretical analysis is also provided in [20]. Active basis can indeed capture the "edge" information in a texture and has the advantage that the direction of edges can also be obtained. Thus, the histogram of active basis can be effectively used for modeling textures with same illumination azimuthal angles, as different angles can dramatically change the appearance of 3D surface texture [11]. This can be clearly seen in Fig. 8. Texture images in the same class captured under different illumination angles have different base histograms. However, when illuminated under same illumination azimuthal angles, these textures tend to produce similar base histograms. Thus, we can estimate the illumination direction of a 3D surface texture by examining similarities between base histograms. 


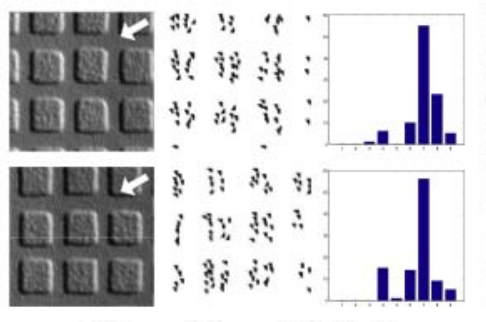

(a) $\sigma=45^{\circ} \tau=30^{\circ} \mathrm{N}=9$

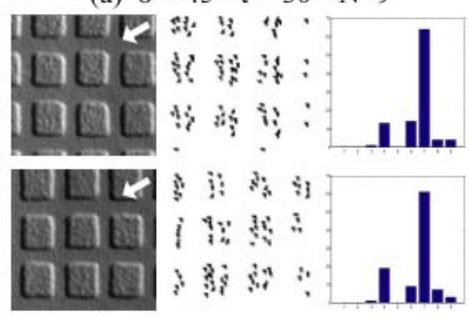

(b) $\sigma=60^{\circ} \tau=30^{\circ} \mathrm{N}=9$

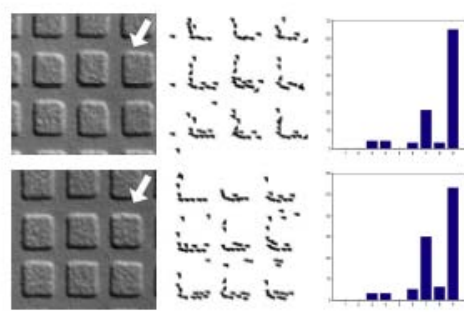

(c) $\sigma=45^{\circ} \tau=60^{\circ} \mathrm{N}=9$

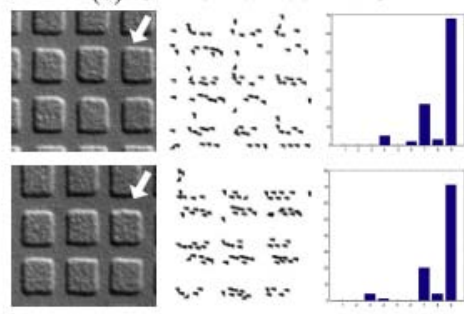

(d) $\sigma=60^{\circ} \tau=60^{\circ} \mathrm{N}=9$

Fig. 8. The base models and histograms of texture "acd" [10]. The texture images are the patches of Label "acd" taken at illumination zenithal angles of $\sigma$ and azimuthal angles of $\mathrm{T}$ (indicated by block arrows).

\section{Estimating Illumination Directions}

Once a set of texture samples having the most similar texture orientation to the test sample are found, we estimate the illumination direction by finding the best match based on the correlation coefficients of active base histograms. The correlation coefficient between base histograms is defined as

$$
\operatorname{corrcoef}(X, Y)=\frac{\sum_{i=1}^{N}\left(X_{i}-\bar{X}\right)\left(Y_{i}-\bar{Y}\right)}{\sqrt{\sum_{i=1}^{N}\left(X_{i}-\bar{X}\right)} \sqrt{\sum_{i=1}^{N}\left(Y_{i}-\bar{Y}\right)}}
$$

Where $X$ is the histogram vector of the test image, $Y$ is the histogram vector of a training image, and $N$ is the number of Haar orientations.

The illumination direction of the test texture is determined by the sample whose base histogram has the largest correlation coefficient to the test one. Euclidean distance was also 
tested to measure the similarities between histograms, but it exhibited lower performances. In the next section, we provide a comparison of the accuracy for both the Euclidean distance and the correlation coefficient.

\section{Experimental Results}

The main goal of the proposed method is to correctly estimate illuminated directions using textures in the database. The Mojette transform is used to compute the texture orientations, according to which the base model will then be compared so that the illumination direction can be determined. We first show the performances of the Mojette transform on estimating texture orientations and then present the illumination estimation results.

\section{Results of Estimating Texture Orientations}

We first evaluate the Mojette transform on estimating texture orientations. We use a data set consisting of 15 surface textures of size $512 \times 512$ from the PhoTex texture database [10], which will be used in later experiments for estimating illumination direction. We did not select the Brodaz database in our experiments, because there is no known illumination information available.

The experiments are designed as described in [13]. Each texture image is divided into four $256 \times 256$ non-overlapping regions. To create the test set, each $256 \times 256$ region was rotated at angles $10^{\circ}$ to $160^{\circ}$ with $10^{\circ}$ increments and from each rotated image one $128 \times 128$ subimage was selected. With this approach, we created a total number of $960(15 \times 4 \times 16)$ images for the testing set.

We then calculate the Mojette projections for angles $0^{\circ}$ to $180^{\circ}$ with $2.5^{\circ}$ increments and then threshold the second-order derivative vector $V^{\prime \prime}$ to determine the texture orientation. The 
error is defined as the difference between the estimated orientations of each two successive rotated textures (which are supposed to be $10^{\circ}$ ) minus 10 . Fig. 9 shows the error mean and squared root of mean square (SRMS) error of the direction estimation for each texture in the testing set. The comparison with results in [13] is also shown in Fig. 9. It can be seen that our method for estimating texture orientations using the Mojette transform outperforms traditional methods based on the Radon transform in [13].

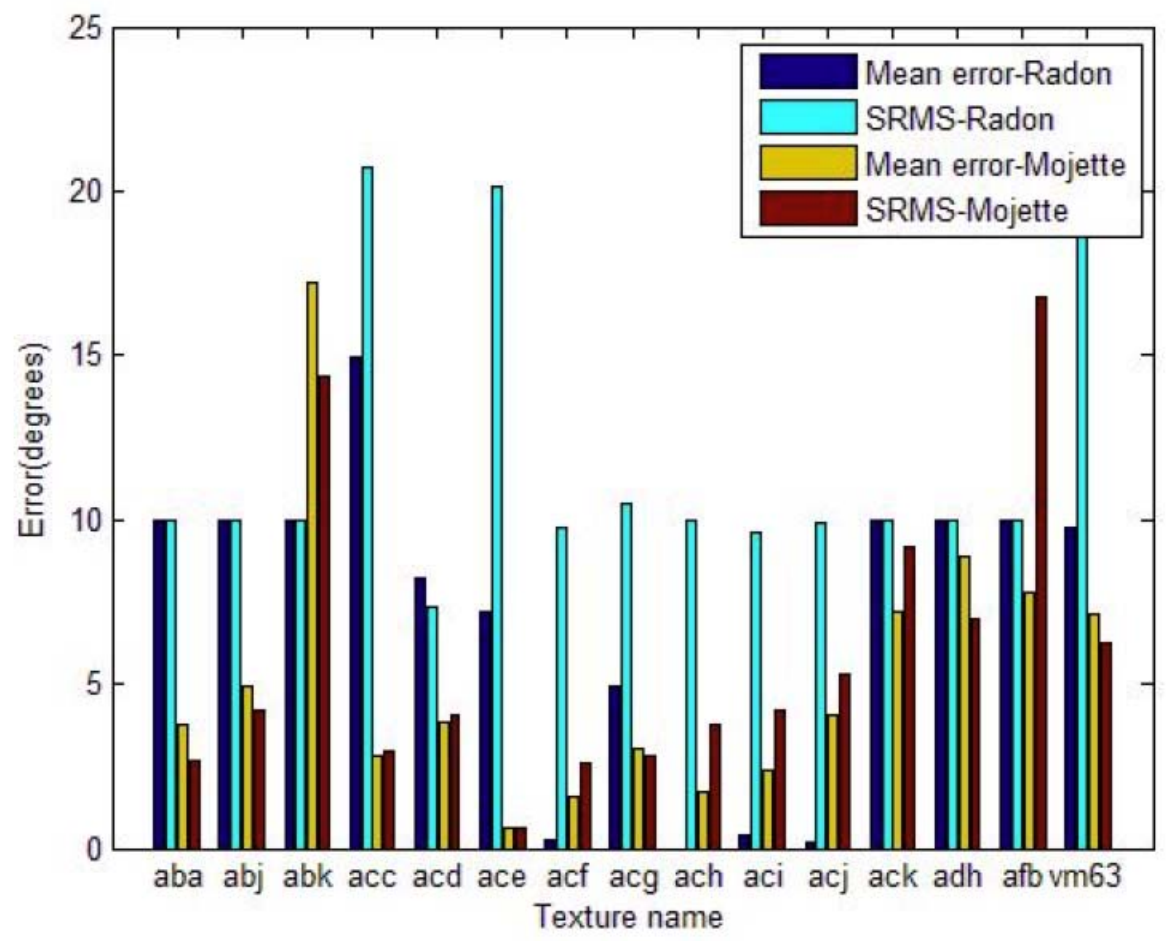

Fig. 9.Comparison of performance of our method and the one in [13] for texture orientation estimation.

Although our approach is proposed for estimating anisotropic texture orientations and illumination directions, which are difficult subjects for previous methods [8], we also perform an experiment to verify the performance of the Mojette Transform on isotropic textures. We have witnessed on Fig. 4 that the Mojette projections have a significantly lower variance for an isotropic texture (Fig. 4(b)) than for a anisotropic texture (Fig. 4(a)). However, in the following 
experiment we are interested in studying the behavior of the proposed method for a locally oriented texture, such as a circular pattern. For such locally oriented textures, the Mojette transform may exhibit some flat projections. Fig. 10 shows an example. In this experiment, 12 image blocks with size of $64 \times 64$ in different directions are selected. The Mojette Transform was computed within every block and the variance of the projections at different angles on each block was calculated. The results clearly show that for every image block there is a strong peak (corresponding to texture orientation), whereas the projection variance of the whole texture has no obvious peak and our model outputs "no direction". This is exactly the same as what human observers can perceive.

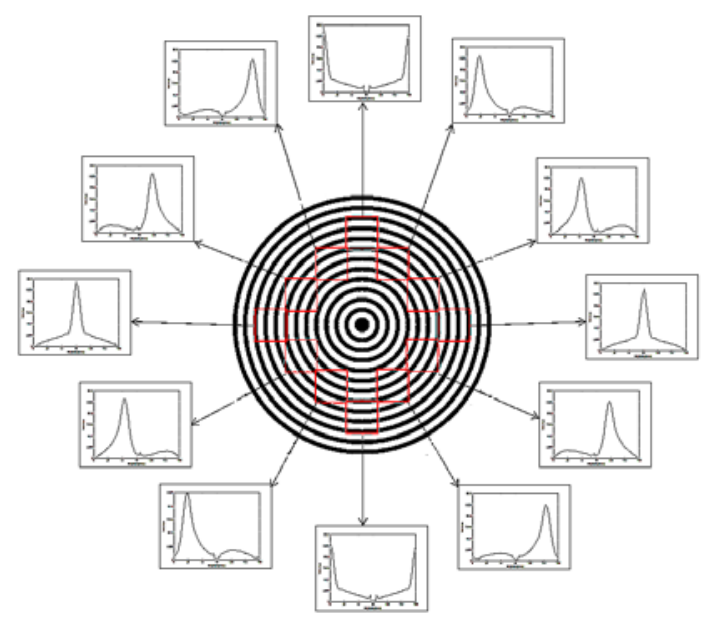

(a)

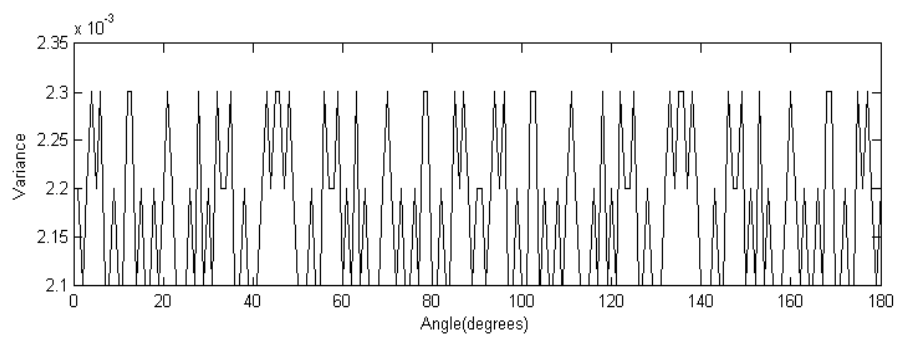

(b)

Fig. 10.(a) The variance of projections at different angles on each block. (b)The variance of all projections on the whole image is also shown for comparison.

\section{Results of Estimating Illumination Directions}

The proposed method can not only estimate the illumination direction of a texture in the training data set, but also those with similar texture orientations yet excluded from the training set. We compare the Euclidean distances between vector $V^{\prime \prime}$ to measure the similarity of the 
orientations between textures. Then we can compare the base histograms of the pair with the most similar for illumination direction estimation.

In our experiment, we exploit the PhoTex texture database [10], which contains many sample textures. Each texture is represented using 36 images captured under a variety of different illumination conditions. The illumination angle is denoted by a slant (zenith) angle and a tilt (azimuth) angle. We select 15 texture images from the PhoTex texture database [10] for our experiments. Each image has been imaged under zenithal angles of 45 and 60, with azimuth varying between $0^{\circ}$ and $150^{\circ}$. The size of all images is $512 \times 512$. We divided each texture image into four $256 \times 256$ quarters: one is used to compose the training set, while the other three compose the test set. Fig. 11 shows one image of each sample we used in the experiment.

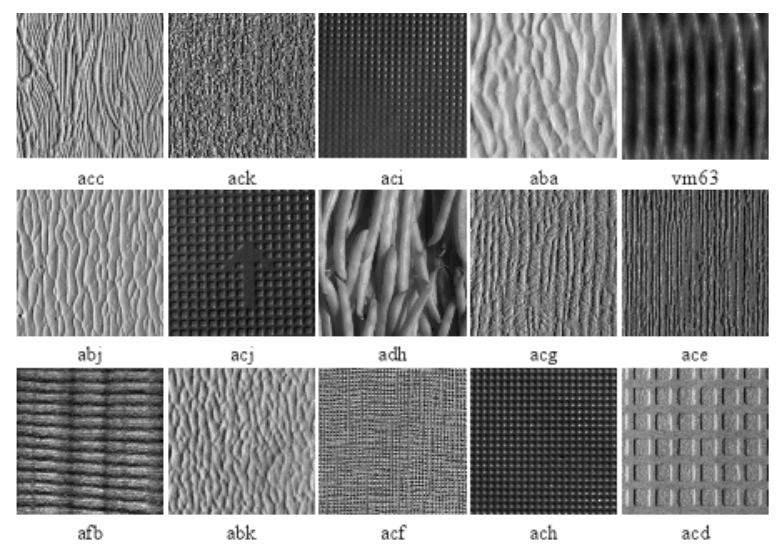

Fig. 11.15 Surface textures within the PhoTex texture database [10].

We demonstrate the effectiveness of the proposed approach in two groups of experiments. In the first group of experiments, all textures are used to assess how well the method can identify the texture directions and estimate the illumination for a test sample whose texture class is included in the training set. We first test how the parameters used for learning active bases can affect the final estimation accuracy. We used different combination of three parameters, i.e. the number of orientations $(N)$, the size of Haar feature (haarSize) and the 
number of bases (baseNumber). Fig. 12 shows the results. It can be seen that the most accurate estimation result is obtained when using $N=12$, harrSize $=13 \times 13$, and baseNumber $=300$. The figure also provides a comparison of the performances between the Euclidean distance and the correlation coefficients. The correlation coefficient outperforms the Euclidean distance in this case.

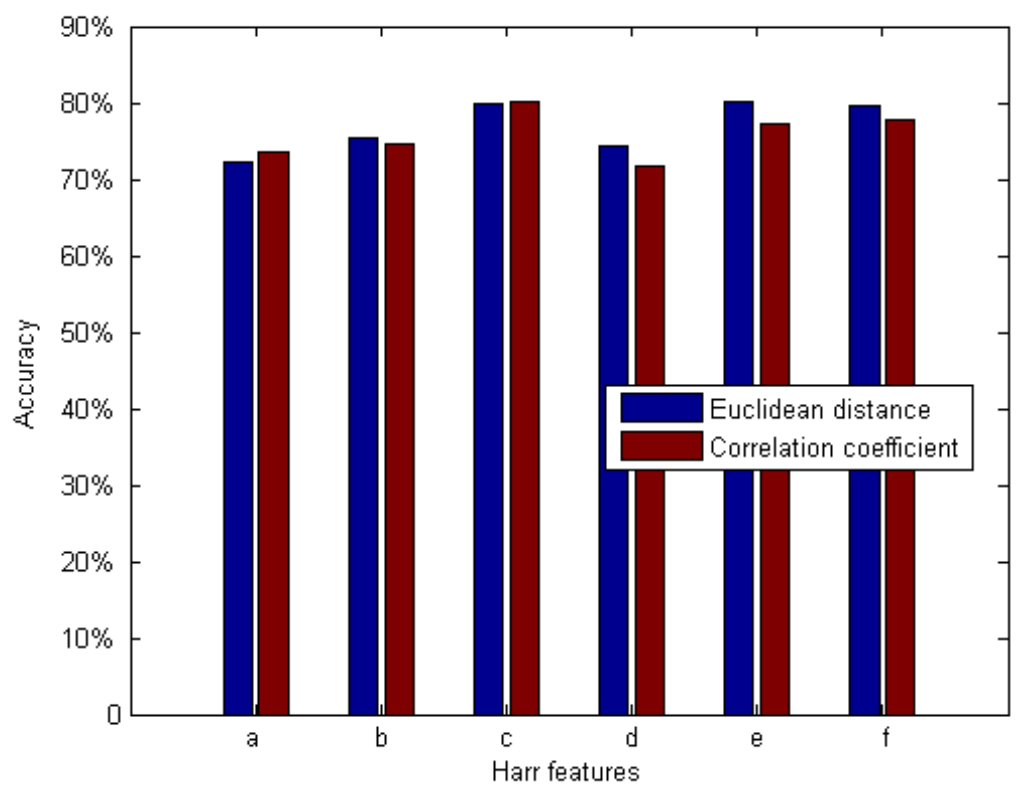

Fig. 12.Comparison of the accuracy of illumination direction estimation based different similarity metrics (correlation coefficients and Euclidean distance) and different combinations of parameters used in Haar functions. On the x-axis: $a: N=12$, haarSize $=9 \times 9$, baseNumber $=200 ; b: N=12$, haarSize $=9 \times 9$, baseNumber $=300 ; c: N=12$, haarSize $=13 \times 13$, baseNumber $=300 ; d: N=15$, haarSize $=9 \times 9$, baseNumber =200; e: $N=15$, haarSize=9x9, baseNumber $=400 ; f: N=15$, haarSize $=13 \times 13$, baseNumber $r=300$.

The threshold, determining how many textures with similar Mojette projection vectors to the test sample should be used for comparing active base histogram, can also affect the estimation accuracy. In our experiments, we have tested a number of values using the closest distance to the test sample's $V^{\prime \prime}$ as the metric. From Fig. 13, a threshold of 146 appears to give the best result. In this case, we also used the best parameter combination for Haar features, i.e. $N=12$, harrSize $=13 \times 13$, and baseNumber $=300$. 


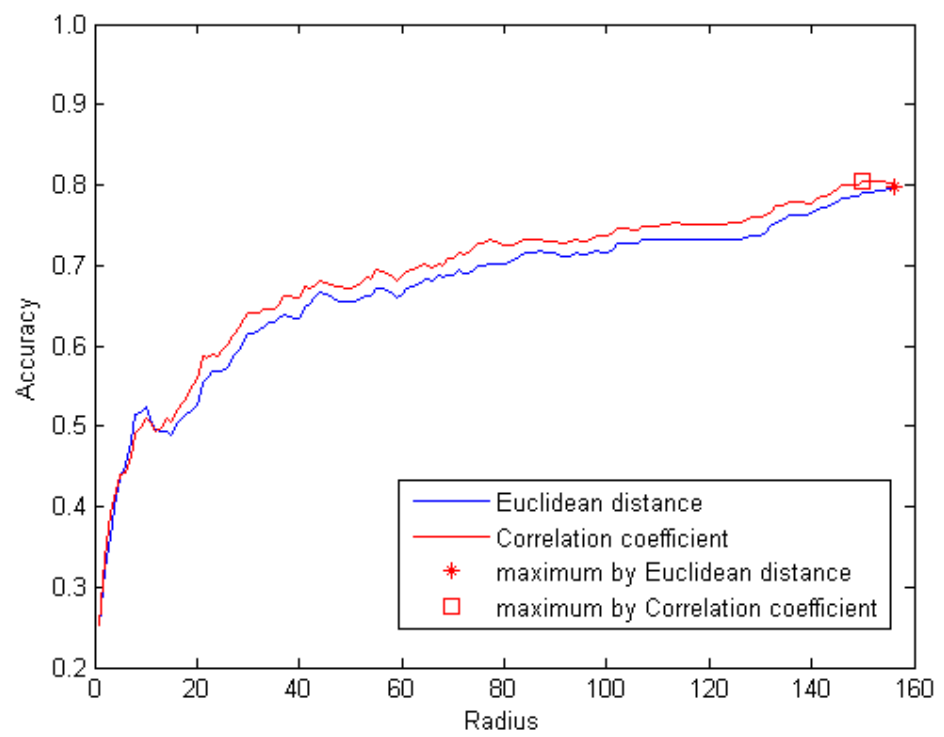

Fig. 13.Comparison of different thresholds of the number of textures used for comparing base histogram. The $\mathrm{x}$-axis represents the number of textures that have similar Mojette projection feature vector $\mathrm{V}$ " to that of the test sample.

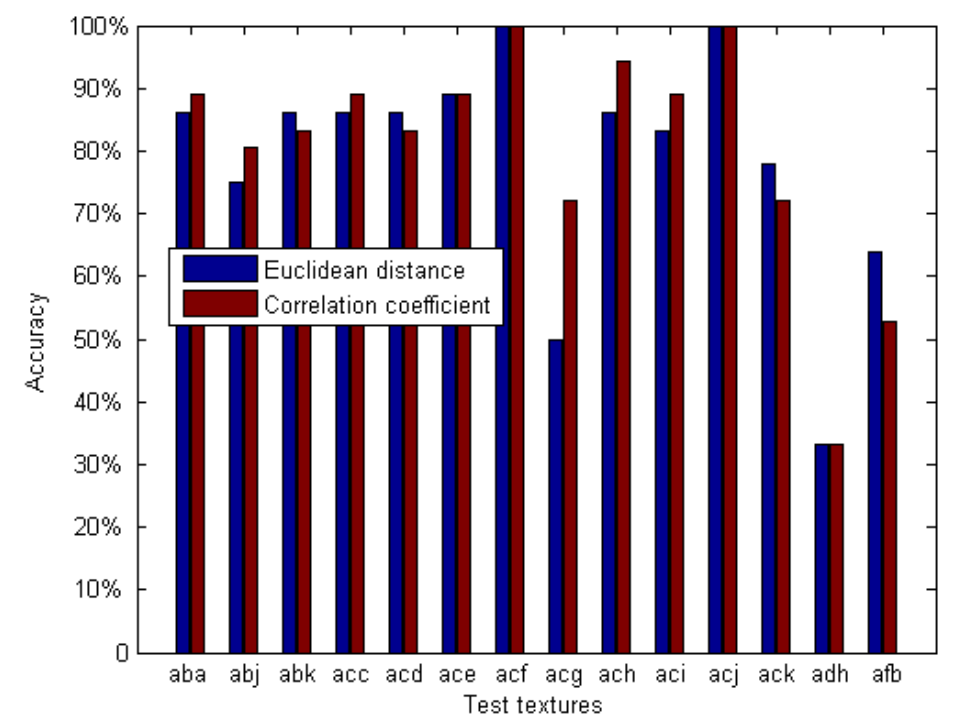

Fig. 14. The accuracy of illumination direction estimation for each texture class when $\mathrm{N}=12$, haarSize $=13 \times 13$ and baseNumber $=300$ are used for learning active basis.

Fig. 14 shows the accuracy of illumination direction estimation when the best combination of parameters is used for obtaining active basis. It can be seen that texture "adh" produced the worst result. This is because texture "adh" consists of green beans (see Fig. 11) and the surface height has large variations. They lead to large area of cast shadows when illumination 
directions are changed. Since shadows are dark and make normal pixel values become smaller, varying illumination directions does not change the texture appearances as normal. Accordingly the accuracy of illumination direction estimation is reduced.

While the first group of experiments shows that our method can be effectively used to estimate the illumination direction of a test sample whose texture class is included in the training set, we also wish to know the performance of our method in the case that the test texture class is not included in the training set. This is also a novel point in the paper, as traditional methods in the literature did not consider this case. Thus, we carry out the second group of experiments, which is based on a "leaving-one-out" strategy. Among 15 surface textures showed in Fig. 15, we always use one texture as the test while the remaining ones are treated as the training set. We first utilize the Mojette transform to obtain the orientations of test image. Then the base histogram of the test image is compared with the surface texture having the most similar texture orientations in the training set.

In this experiment, for texture images with a size of $512 \times 512$, we used a size of $13 \times 13$ for the Haar function and fixed a total number of 400 bases for the active sketch algorithm. The orientation takes $N=12$ equally spaced angles in $[0, \pi]$. The shift along the normal direction is $\{-2,0,2\}$ pixels. The shift of orientation is $\{-1,0,1\}$ angles out of 9 angles. 


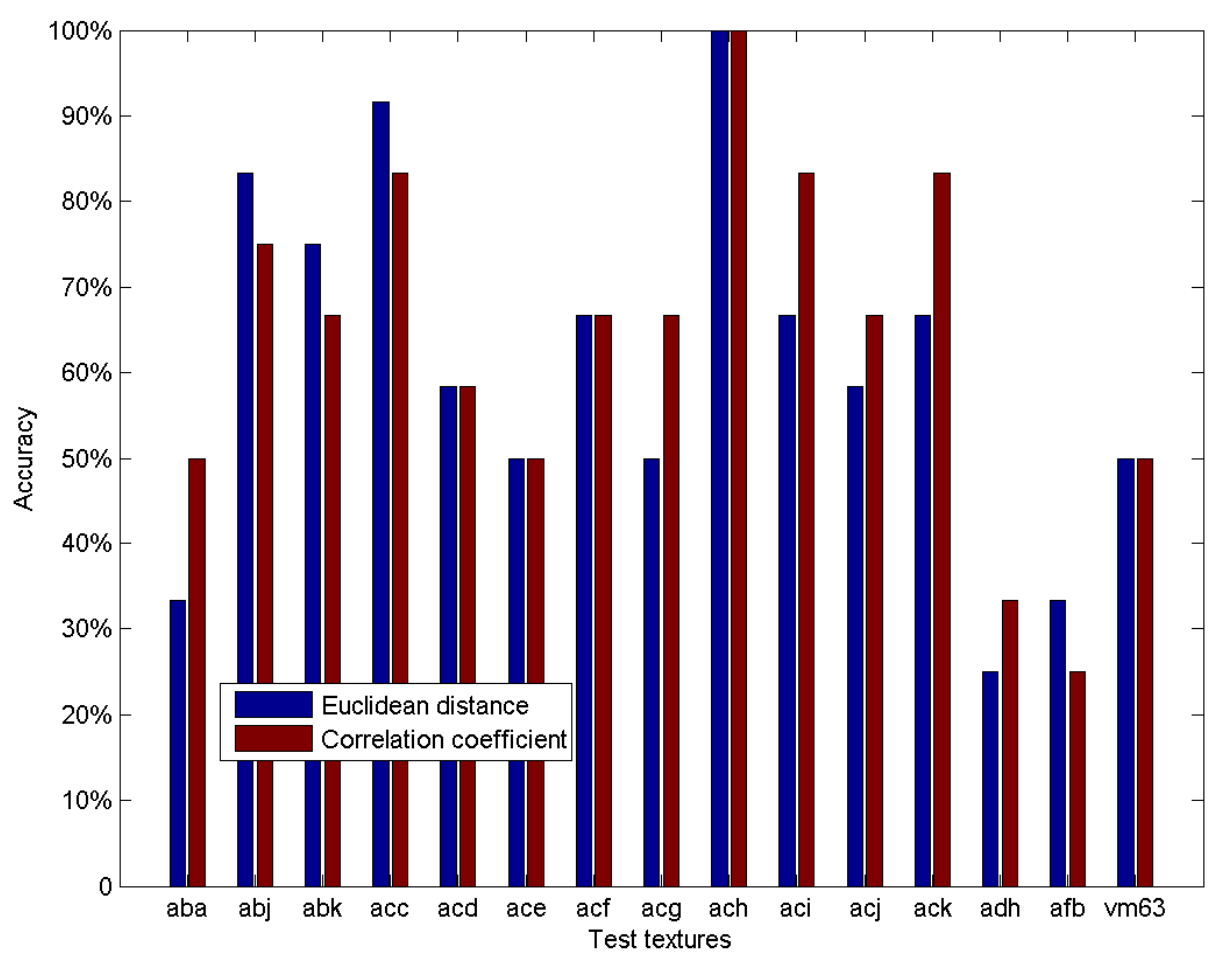

Fig. 15. The accuracy of illumination direction estimation when a test texture class is not included in the training set.

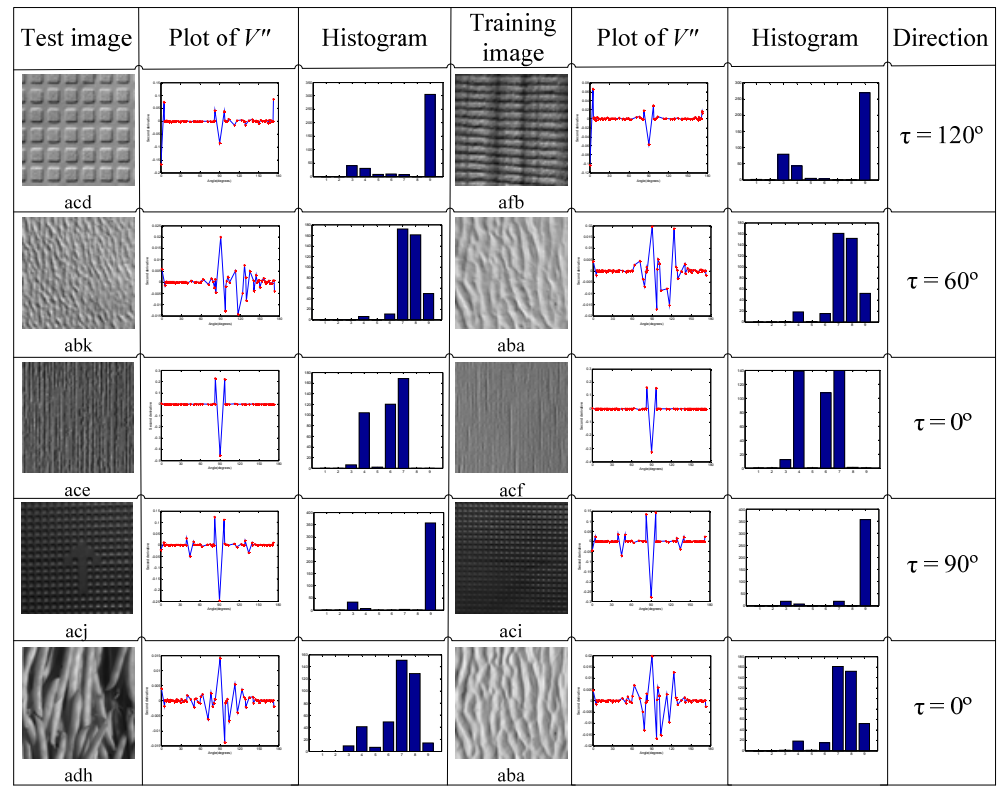

Fig. 16. Examples of illumination direction estimation results. From left to right are: the test images, their texture orientations, the base histograms, the training images, along with their texture orientations, their base histograms and the estimated direction. 
Fig. 15 shows the estimation accuracy. Texture "adh" and "afb" produced the worst result, and we can also see that the textures that are not similar to any other in the training set produced relatively low results. However, the method can correctly estimate the illumination directions of some textures that have similar orientations with those in the training set. Though the data set is limited, the illumination of the test image can be effectively estimated once a number of textures with similar orientations are found in the database. Fig. 16 shows the examples of matched Mojette projection features and base histograms.

Compared with the method proposed in [12], a significant difference between the two methods is that our approach can estimate illumination directions of a texture that is not included in the training set, while the one in [12] cannot. Even in the case of test textures that are included in the training set, the estimation accuracy of our method is $80.3 \%$, while the one of the method [12] is $76 \%$ within 5 degrees of error value and $82 \%$ within 10 degree of error value. Thus, our method does have advantage over the one in [12].

\section{Conclusions}

We introduced a new method for estimating illumination directions of 3D surface textures. In this study, the sample texture directions are first estimated using Mojette transform, and then the Active Basis method is employed to extract texture features and generate a base model, from which the base histograms are computed. Finally, the histogram of the learned model is compared with those of the textures having the most similar texture orientations in the training data set. The proposed method is tested using a variety of $3 \mathrm{D}$ surface textures and the experiments produced convincing results. Differing from previous works, the illumination direction of a test texture that is not included in the training set can also be estimated. 
It should be noted that the illumination directions of certain surface textures has not been effectively estimated in our experiments. For some anisotropic surfaces, when the illumination direction is aligned with the surface orientation (e.g., at the angle of $120^{\circ}$ for texture "acc" and "aba", $90^{\circ}$ for "abk", angles of $0^{\circ}$ and $90^{\circ}$ for "acd" and "acf"), the estimation error tends to be

larger. This error is most probably due to the weak response of the structure texture to certain illuminations directions. For the Mojette transform, in practice, the angular energy distribution may change significantly in a single texture. Therefore, it is not always reliable to find the dominant orientation using the contributions of all frequencies. Future work may further investigate other features based on active basis and relevant similarity measurements to improve the estimation accuracy.

\section{Acknowledgement}

This work was supported by grants from the National Natural Science Foundation of China (No. 60702014 and No. 40976002). We also would like to thank anonymous reviewers and Dr. Gerald McGunnigle for their helpful suggestions on the presentation of this paper.

\section{References}

1. Y. N. Wu, Z. Si, C. Fleming, S.-C. Zhu. Deformable template as active basis. ICCV, 2007.

2. Ying Nian $\mathrm{Wu}$, Zhangzhang Si, Haifeng Gong and Song-Chun Zhu. Learning Active Basis Model for Object Detection and Recognition. International Journal of Computer Vision, 2009.

3. Lai, Bin; Zhang, Deng-Yi; Qu, Cheng-Zhang. Object tracking by using active basis model. Proceedings of SPIE, 2009. 
4. Y. N. Wu, Z. Si, H. Gong, and S.-C. Zhu. Active basis model, shared sketch algorithm, and sum-max maps. IJCV, 2008.

5. Q. Zheng, R. Chellappa. Estimation of illuminant direction, albedo and shape from shading. IEEE PAMI, 13(7):680-702, 1991.

6. P. Nillius, J-O. Eklundh. Automatic estimation of the projected light source direction. In Proc. CVPR, volume 1, pages 1076-1083, 2001.

7. J. J. Koenderink, S. C. Pont. Irradiation direction from texture. J. of the Optical Society of America, 20(10):1875-1882, 2003.

8. Manik Varma, Andrew Zisserman. Estimating Illumination Direction from Textured Images. In Proc. CVPR, Volume 1, pages.179-186, 2004.

9. Karlsson, Stefan; Pont, S.C.; Koenderink, J.J. "Illuminance flow over anisotropic surfaces" Journal of the Optical Society of America. A, Optics, image science, and vision, Volume: 25, Issue: 2 (2008), pp. 282-291.

10. PhoTex database. Texture lab, Heriot-Watt University, Edinburgh, UK. Available on-line http://www.macs.hw.ac.uk/texturelab/resources/databases/photex/.

11. M. Chantler, G. McGunnigle, A. Penirschke, and M. Petrou. Estimating lighting direction and classifying textures. In Proc. BMVC., pages 737-746, 2002.

12. M. Chantler, M. Petrou, A. Penirsche, M. Schmidt, G. McGunnigle. Classifying Surface Texture while Simultaneously Estimating Illumination Direction. International Journal of Computer Vision, Volume 62, Issue 1-2, Pages: 83 - 962005.

13. K. Jafari-Khouzani and H. Soltanian-Zadeh. Radon transform orientation estimation for rotation invariant texture analysis. IEEE Transactions on Pattern Analysis and Machine Intelligence, 27(6):1004-1008, 2005. 
14. Jia P., Dong J., Qi L., Autrusseau F., "Directionality Measurement and Illumination Estimation of 3D Surface Textures by Using Mojette Transform", International Conference on Pattern Recognition, Tampa, FL, USA, 2008-12, IAPR, December 2008, To appear.

15. J. Radon, "Uber die Bestimmung von Functionen durch ihre Integralwerte langs gewisser Mannigfaltigkeiten”, Berichte Sachsische Academie der Wissenchaften, Leipzig, Math. Phys. K1, vol 69 pp. 262-267, 1917.

16. A. Kingston, B. Parrein, F. Autrusseau, "Redundant Image Representation via Multi-Scale Digital Radon Projection", IEEE International Conference on Image Processing, ICIP'2008, pp. 2920-2924, Oct12-15, 2008, San Diego, California, USA.

17. A. Kingston, F. Autrusseau, "Lossless Image Compression via Predictive Coding of Discrete Radon Projections", Elsevier Signal Processing: Image Communication, Vol 23/4 pp 313324, May 2008.

18. Paul Viola and Michael J. Jones. Rapid Object Detection using a Boosted Cascade of Simple Features. Proc. of IEEE Conference on CVPR. 2001(1):511-518.

19. JP. Guédon, N. Normand, Spline Mojette Transform. Applications in Tomography and Communications. In Proc. 11th European Signal Processing Conference, Vol. 2 (Sep 2002), pp. $407-10$.

20. Vaidyanathan, G.; Lynch, P.M.; Texture direction analysis using edge counts. Southeastcon '89. Proceedings. Energy and Information Technologies in the Southeast., IEEE. 9-12 Apr 1989. page(s): $733-738$ vol.2.

21. B. Eswara Reddy, A. Nagaraja Rao, A. Suresh, V. Vijaya Kumar. Texture Classification by Simple Patterns on Edge Direction Movements. IJCSNS International Journal of Computer Science and Network Security, VOL.7 No.11, November 2007 